\title{
Enigma of Fixed Retainers
}

\author{
Dr Jijo C Eapen,' Dr Amit Prakash² \\ 'Practitioner Orthodontist, ${ }^{2}$ Reader, People's College of Dental Sciences, Bhopal, India \\ Correspondence: Dr Amit Prakash; Email: amitprakashzo@gmail.com
}

\section{ABSTRACT}

Fixed retainers are a reliable form of retention for avoiding relapse and maintaining dental arch shape. They function regardless of patient cooperation, and they fulfill high esthetic expectations. Teeth have tendency to return to their former position, as time required for retaining the treatment result. The most appropriate mode of retention for the post-treatment situation should be used, based on a careful evaluation of the patient's pretreatment diagnostic records, habits, cooperation, growth pattern, and age. Retention after orthodontic intervention is as important part of the therapy as the active treatment. Age and maturity of the patients, result of the orthodontic intervention, origin and character of the anomaly, type of the retainer, compliance of the patients; all can influence the chance of relapse. The retention period should be twice longer than the active orthodontic treatment. This article covers various aspect of fixed retention in detail.

Keywords: fixed retainer, orthodontics, relapse, stability

\section{INTRODUCTION}

Retention has been defined by Moyers as, "The holding of teeth following orthodontic treatment in the treated position for the period of time necessary for the maintenance of the result". ' The time of retention varies according to the age of the patient, occlusion gained, tooth movements accomplished, length of cusps, health of the tissues etc; from a few days, to a year or two years, or often longer. The close relationship between active orthodontic treatment and retention was emphasized by Hellman; who said that "retention is not a separate problem in orthodontics, but is a continuation of what we are doing during the treatment." 2

Knierim was the first to report the use of bonded fixed retainers. Zachrisson reported about the possible benefits of using multi-stranded wires instead of the earlier use of round orthodontic wire for constructing the bonded retainers. The proponents of multi-stranded wire claim advantages like increased mechanical retention for composite with no need of retentive loops and allowance of physiologic movement of teeth in spite of bonding several adjacent teeth due to its flexibility. As an alternative to multistranded wire, the uses of resin fiberglass strips have been developed.,4 Long-term stability after orthodontic treatment has been found to be unpredictable at the individual level as growth and dental tissue changes may interfere with an otherwise good treatment result. Research has shown that the periodontal ligament needs 3-4 months to remodel. The collagen fiber network needs 4-6 months to reorganize, and the elastic supra-crestal fibers need up to one year to settle. ${ }^{1,3}$ Because of these factors, relapse tendency is highest immediately after debonding and in the first 12 months post-treatment. 5,6

\section{DISCUSSION}

\section{Why fixed retention?}

Following orthodontic treatment, a reduction in arch length and inter-canine width is evident. Inter-molar width, if expanded during treatment; tends to return toward the pre-treatment value. 7 Treated cases should be viewed as dynamic and constantly changing, at least through the third and fourth decade and perhaps throughout the life. For these reasons, orthodontists are turning towards permanent retention to ensure stability of post-treatment tooth positions.8.9 The biologic aims to be considered when choosing a retainer are maintenance of periodontal support, maintenance of optimal oral hygiene and maintenance of functional forces on the teeth. ${ }^{2}$ The term "differential retention" implies that special attention given to the site that is most prone to relapse in each orthodontic case.10 Adjunctive procedures such as interproximal stripping 
and circumferential supra-crestal fibrotomy can also be applied to the teeth or surrounding periodontium to aid the retention process. 3,9 Extended retention periods as long as ten years are now recommended by most clinicians. ${ }^{11}$

\section{Generations of fixed retainers}

Bonded fixed retainers consist of a length of orthodontic wire bonded to the teeth with acid-etch retained composite. At present following three generations of fixed retainers are available: ${ }^{12}$

First generation: Plain blue Elgiloy wire with a loop at each terminal end (Figure 1)

Second generation: Similar diameter but multistranded wire used (Figure 2)

Third generation: Round 0.032" stainless steel or 0.030" gold coated wire (Figure 3).

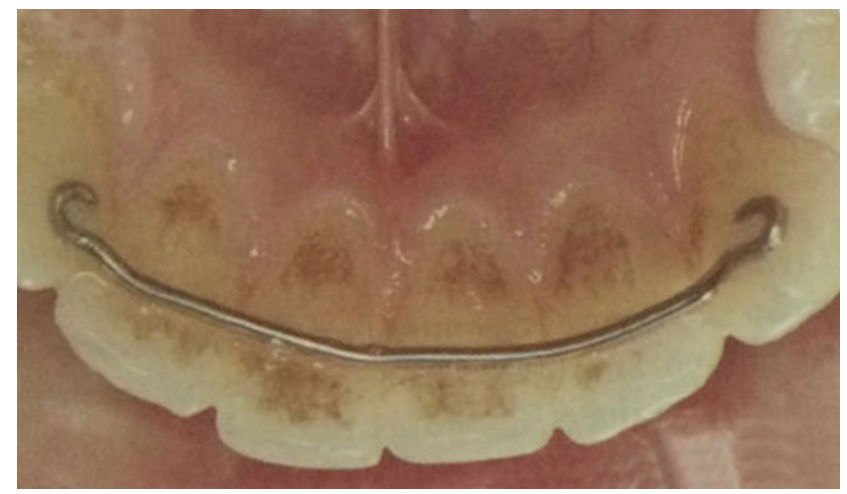

Figure 1: First generation bonded retainer

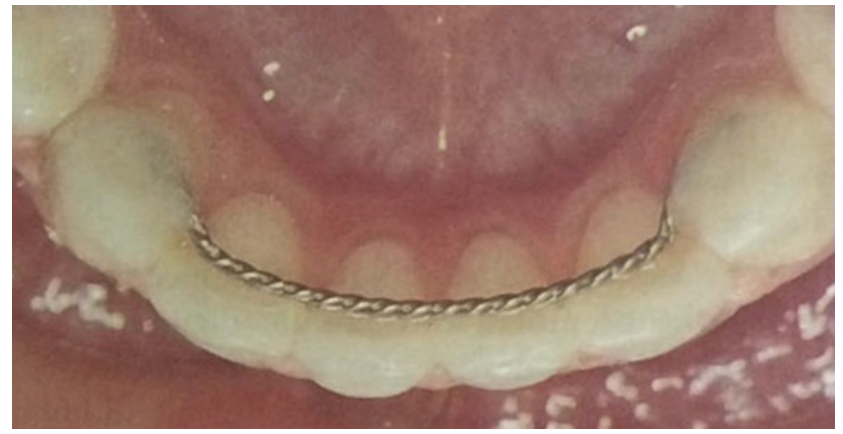

Figure 2: Second generation bonded retainer

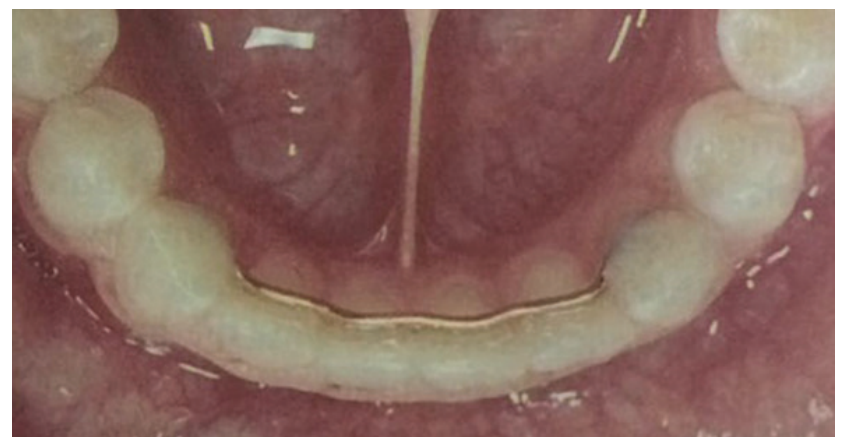

Figure 3: Third generation bonded retainer

\section{Indications}

Indications for placement of a bonded canine-tocanine retainer includes severe pretreatment lower incisor crowding or rotation, planned alteration in the lower inter-canine width, after advancement of the lower incisors during active treatment, after nonextraction treatment in mildly crowded cases and after correction of deep overbite. ${ }^{4}$

Zachrisson listed the following indications for clinical use of flexible wire retainer:

1. Closed median diastema

2. Spaced anterior teeth

3. Adult cases with potential post-orthodontic tooth migration

4. Accidental loss of maxillary incisors requiring closure and retention of large anterior space

5. Spacing reopening, after mandibular incisor extractions

6. Severely rotated maxillary incisors

7. Palatally impacted canines

\section{Advantages}

The main advantage of fixed retainer as compared to removable retainer is that they are invisible, are welltolerated by patients and are virtually compliancefree.1,13 Artun concluded from his investigation that long-term use of bonded retainers caused no damage to the teeth and to the hard and soft tissues adjacent to the wire. ${ }^{14}$

\section{Disadvantages}

Fixed retainer placement is time-consuming and technique-sensitive, and for some individuals they can be difficult to maintain, encouraging plaque and calculus accumulation. Additionally, proclination of mandibular incisors induced by treatment has been linked to decreased attachment levels, contributing to recession. Even though this hypothesis has not been unanimously accepted, there is a possibility that proclined mandibular incisors retained with a fixed bonded appliance for long period of time may cause attachment loss. ${ }^{14}$

\section{Methods of stabilizing fixed retainers}

Different methods of stabilizing wire includes timesaving fixed lingual retainer using DuraLay resin transfer," acrylic transfer tray for direct-bonded lingual retainers, ${ }^{12} \mathrm{~W}$-shaped lingual retainer wire stabilizer, ${ }^{15}$ 
modified Kesling's separators for stabilizing lingual retainer wire, ${ }^{16}$ Wire Jigs for stabilizing lingual retainers, ${ }^{17}$ Retainer Positioner, ${ }^{18}$ Stabilizing Springs for fixed lingual retainer, ${ }^{19}$ lingual retainer stabilized by Modifying Archwire, ${ }^{20}$ indirect method of fixing lingual retainer using addition polysilicone tray ${ }^{21}$ and an indirect method of fabrication of lingual bonded retainer. ${ }^{22}$

\section{Success and failure rates}

Reports show $67 \%$ of the achieved orthodontic treatment result maintained after 10 years postretention. About half of the total relapse takes place in the first 2 years after retention. All occlusal traits relapsed gradually over time but remained stable from 5 years post-retention with the exception of the lower anterior contact point displacement. ${ }^{15}$ Failure rates reported for bonded retainers ranged from 10.3-47.0\%. The failure rate amongst bonded retainers was $22.9 \%$ and the majority of failures occurred during the third year of observation. ${ }^{15}$ Dahl and Zachrisson reported low failure rates for flexible spiral wire retainers (FSWR) for five stranded wires than for other wire types. The most common causes of these failures were debonding $(37.5 \%)$, fracture plus debonding $(1.4 \%)$, and fracture (0.7\%). ${ }^{10,23}$ The difference may be explained by technical factors such as the use of adequate composite resin over the wire, smooth contouring of the adhesive resin, completely undisturbed setting of the adhesive in every case, and careful adaptation of the wire to the lingual contours of the teeth, as well as avoidance of occlusal interference from opposing teeth. ${ }^{8}$ The failure rate is approximately twice as great in the maxilla as the mandible, and this is most likely because of occlusal factors. The most common site of failure is at the wire/composite interface. The use of increased bulk of composites or materials of greater abrasion resistance may improve the longevity of the retainer. ${ }^{4}$ Insufficient patient care seems to be a possible reason for the repeated bonding. ${ }^{23,24}$

\section{Bonding materials}

The composite resin should be easily manipulated and have sufficient strength to hold the retainer wire and sufficient abrasion resistance to not significantly abrade away with normal dietary mastication. Some authors recommend the use of Concise orthodontic resin (3M Unitek), a self-curing composite resin, or a combination of light-cured resin with a concise overlay. In one study of multiple composite resins, including a posterior restorative composite, the authors concluded that Transbond (3M Unitek), a light-cured resin and Concise were both acceptable for bonding retainer wires because of their strength and their relatively high abrasion resistance. In that same study, the authors cautioned against diluting the composite resin too much with the unfilled resin or sealant because it decreases the wear resistance of the composite and may lead to premature retainer failure due to composite wear. Treating the wire surface before bonding can also strengthen the bond at the wire-composite interface. Microetching or sandblasting with aluminum oxide has been claimed to enhance the bond between metal and composite resin by at least $300 \%$. Claims have also been made that adhesion promoters such as silane or other commercially available adhesion promoters can increase the metal composite bond strength. Strengthening the bond between the retainer wire and the composite resin would strengthen the retainer and decrease clinical failure rates, which have proven to be the primary disadvantage of bonded retainers. ${ }^{\text {? }}$

\section{Wires used in fixed retainers}

In 1983, Zachrisson introduced flexible spiral wire retainers (FSWRs). These retainers use a multistranded wire and include all anterior teeth. The flexibility of the wire reduces the concentration of stress within the bonding composite, thus minimizing the probability of subsequent failure. Baysal et al tested two types of dead-soft wires and a commonly used fie-stranded stainless steel wire. Bearn recommended 0.0215-inch multistranded wire for fabricating FSWRs. Aldrees et al found greater bond strength values with coaxial wire (PentaOne) compared to a solid chain retainer. They also stated that the flattened wire increases patient comfort. The coaxial wire is recommended as an initial arch wire because it applies light and gentle force. This wire is very flexible and possesses great spring-back characteristics. Manufacturers recommend dead soft respond for bonded lingual retainers. According to Cooke and Sherriff, when a vertical force is applied to a bonded wire, tension, shear, and torsion forces may occur simultaneously. Lumsden et al reported an increase in wire fractures as retainers aged. ${ }^{15}$ While this flexibility may appear to be advantageous, Zachrisson ${ }^{25}$ reported that the wire fracture incidence decreases as wire diameter increases.

\section{Fixed retention procedure}

The direct technique requires a length of wire to be prefabricated to accurately fit a recent cast. Loops are not required at the ends of the wire. The adaptation of the wire is checked clinically to ensure it locates passively against all tooth surfaces to be retained. The teeth are subsequently pumiced and acid etched for 
direct bonding of orthodontic attachments. The wire is then accurately located on the teeth. At this point authors differ in their approach, and many methods for locating the wire have been described. These include the use of dental floss, orthodontic elastics, wire ligatures, wires tack welded to the retainer wire localizing devices, or fingers. It is recommended that a small amount of composite be used to tack the retainer in place at each end before adding the bulk of material. The composite can be shaped with an instrument dipped in unfilled resin or alcohol to produce the desired contour. Instruct the patient for proper oral hygiene and use of dental floss beneath the retainer wire and along the mesial contact areas of both canines. Patients are instructed to floss once daily to prevent the accumulation of plaque and calculus. ${ }^{4}$

\section{CONCLUSION}

The ultimate success of a bonded retainer is determined by the size and quality of teeth and the occlusal load on the retainer. As long as the retainer remains intact, the treatment result is maintained, and as long as the patient performs adequate plaque control, no good reason exists to remove it. In general, patients favor permanent bonded retainers over removable retainers for effectiveness, comfort, and impact on quality of life, and they appear to accept the need to wear and maintain the permanent bonded retainers indefinitely. Patients and parents should be informed of the likelihood of post-treatment changes before treatment is undertaken.

\section{REFERENCES}

1. Blake M, Garvey M. T. Rationale for retention following orthodontic treatment. J Can Dent Asso.1998; 64:640-4.

2. Nanda R, Burstone C J. Retention and stability in orthodontics. WB Saunders Company 1993; 1:9-11.

3. Tamilkumaran N, Sumathi F. Fixed Retainers Vs Removable Retainers: Which Is Better? IOSR-J Dent Med Sci. 2013; 11 (6):33-5.

4. Prakash A, Nillachandra, Rai S, Gaurav A, Sandip J. Bonded orthodontic retainers. Indian J Dent Adv. 2012; 4(3):937-40.

5. Bjering R, Birkeland K, Vandevska RV. Anterior tooth alignment: A comparison of orthodontic retention regimens 5 years post-treatment. Angle Orthod. 2015; 85:353-9.

6. Sari Z, Uysal T, Başçiftçi FA, Inan O. Occlusal contact changes with removable and bonded retainers in a 1-year retention period. Angle Orthod 2009; 79(5):867-72.

7. Oesterle LJ, Shellhart WC, Henderson S. Enhancing wire-composite bond strength of bonded retainers with wire surface treatment. Am J Orthod Dentofac Orthop. 2001; 119(6):625-31.

8. Zachrisson BU. Long-term experience with direct-bonded retainers: update and clinical advice. J Clin Orthod 2007; 12(2):728-7.

9. Retief DH, Dreyer CJ, Gavron G. The direct bonding of orthodontic attachments to teeth by means of an epoxy resin adhesive. Am J Orthod Dentofac Orthop. 1970; 58(1):21-40.

10. Miyawaki S, Yasuhara M, Koh Y. Discomfort caused by bonded lingual orthodontic appliances in adult patients as examined by retrospective questionnaire. Am J Orthod Dentofac Orthop. 1999; 115(1):83-8.

11. Shin JL. Time-saving fixed lingual retainer using DuraLay resin transfer. Am J Orthod Dentofac Orthop. 2004; 125:203-5.

12. Esad Zekiç, Gelgör IE. An acrylic transfer tray for direct-bonded lingual retainers. J Clin Orthod. 2004; 38(10):551-3.

13. Baysal A, Uysal T, Gul N, Alan MB, Ramoglu SI. Comparison of three different orthodontic wires for bonded lingual retainer fabrication. Kor J Orthod. 2012; 42(1):39-46.

14. Pandis N. Long-term periodontal status of patients with mandibular lingual fixed retention. Eur J Orthod. 2007; 29:471-6.

15. Schneidera E, Rufb S. Upper bonded retainers Survival and failure rates. Angle Orthod 2011; 81:1050-6.

16. Namit N, Deepali A. Modified Kesling's separators for stabilizing lingual retainer wire. Annals Dent Res. 201 1; 1 (1):96-9.

17. Naik RD, Gandedkar NH. Wire jigs for stabilizing lingual retainers. J Clin Orthod. 2011; 45(5):274.

18. Sahu SK, Jayam BK, Barik AK. Retainer Positioner. J Ind Orthod Soc. 2012; 46(1):53-4.

19. Karthikeyan MK, Prabhakar R, Saravanan R, Rajvikram N, Kuppuchamy. Stabilising springs for fixed lingual retainer. J Clin Diag Res. 2013; 6:1-2.

20. Shreenivas SA. Lingual Retainer Stabilized by Modifying Archwire. J Indian Orthod Soc 2013; 47(4):483-4.

21. Ajithesh KV, Praveen S, Akhter H. Indirect method of fixing lingual retainer using addition polysilicone tray. Unique J Med Dent Sci. 2014; 2(4):103-6.

22. Rajesh D. An Indirect method of fabrication of lingual bonded retainer. Orthod Cyber J. 2013; 1:1-9.

23. Zachrisson BU, Tamer B. Bonding in orthodontics. In: Graber TM, Vanarsdall RL, Xubair Ahmed. Orthodontics Current Principles and Techniques. 5thed. 2012; 21:756-7.

24. Zachrisson BU. Clinical experience with direct-bonded orthodontic retainers. Am J Orthod. 1977; 71 (4):440-8.

25. Zachrisson BU. The bonded lingual retainer and multiple spacing of anterior teeth. Swed Dent J Suppl 1982; 15:247-55. 